\title{
From micro to nano: evolution and impact of drug delivery in treating disease
}

\author{
Jeff Hrkach ${ }^{1} \cdot$ Robert Langer ${ }^{2,3}$ \\ Published online: 8 May 2020 \\ (C) Controlled Release Society 2020
}

\begin{abstract}
Over the past 50 years, drug delivery breakthroughs have enabled the approval of several important medicines. Often, this path starts with innovation from academic collaborations amongst biologists, chemists, and engineers, followed by the formation of a start-up company driving clinical translation and approval. An early wave featured injectable (i.e., intramuscular, subcutaneous) biodegradable polymeric microspheres to control drug release profiles for peptides and small molecules (e.g., Lupron Depot ${ }^{\circledR}$, Risperdal Consta $($ )). With these early successes for microspheres, research shifted to exploring systemic delivery by intravenous injection, which required smaller particle sizes and modified surface properties (e.g., PEGylation) to enable long circulation times. These new innovations resulted in the nanoparticle medicines Doxil ${ }^{\circledR}$ and Abraxane ${ }^{\circledR}$, designed to improve the therapeutic index of cytotoxic cancer agents by decreasing systemic exposure and delivering more drug to tumors. Very recently, the first siRNA lipid nanoparticle medicine, Patisiran (Onpattro $\left.{ }^{\circledR}\right)$, was approved for treating hereditary transthyretin-mediated amyloidosis. In this inspirational note, we will highlight the technological evolution of drug delivery from micro- to nano-, citing some of the approved medicines demonstrating the significant impact of the drug delivery field in treating many diseases.
\end{abstract}

Keywords Drug delivery $\cdot$ Microsphere $\cdot$ Nanoparticle $\cdot$ Controlled release $\cdot$ siRNA $\cdot$ mRNA

\section{Background}

The complexity of human disease biology presents many challenges for the development and approval of new medicines. It is very difficult to design drug molecules with activity specific for a single biological pathway unique to diseased cells; with a pharmacokinetic profile with optimal onset and/or duration of action; or without detrimental side effects due to systemic exposure. In addition, patient compliance in taking medicines can offer significant challenges. Drug delivery systems offer the possibility to design and engineer levels of control beyond

Jeff Hrkach

hrkach@gmail.com

1 Frequency Therapeutics, 19 Presidential Way, Woburn, MA 01801, USA

2 David H. Koch Institute for Integrative Cancer Research, Massachusetts Institute of Technology, 500 Main Street, Cambridge, MA 02139, USA

3 Department of Chemical Engineering, Massachusetts Institute of Technology, 77 Massachusetts Avenue, Cambridge, MA 02139, USA the drug molecule's inherent properties to overcome the aforementioned challenges.

\section{Pioneering drug delivery with biodegradable PLGA microparticles}

Until the mid-1970s, many thought that controlled release drug delivery was limited to small molecules. Pioneering research by Folkman and Langer led the way for sustained release of proteins and other macromolecules [1]. Biodegradable polymers such as the poly(lactide-coglycolide) (PLG) family degrade by hydrolysis in the body and can be engineered to encapsulate drug molecules in microspheres suitable for subcutaneous or intramuscular injection. Modification of polymer molecular weight or composition, drug loading levels, and particle size can be utilized to tune the rate of polymer and microsphere degradation and corresponding drug release profiles. Leuprorelin, or leuprolide, is a gonadotropin-releasing hormone that was initially approved to treat prostate cancer in 1985 in an unencapsulated form and required daily injections due to its inherent pharmacokinetic profile. In 1989, Lupron Depot ${ }^{\circledR}$, a 
PLG microsphere with encapsulated leuprolide acetate, was initially approved for the treatment of prostate cancer as a once-monthly intramuscular injection. This dramatic reduction in the frequency of injections led the way for many additional PLG microsphere-based medicines. Although not a long-term commercial success, in 1999, Nutropin Depot® was approved by the FDA for the treatment of growth failure due to a lack of adequate endogenous growth hormone secretion. This product comprised $22-\mathrm{kD}$ recombinant human growth hormone encapsulated in a PLG microsphere which permitted dosing once or twice a month, as opposed to the typical daily subcutaneous injection dosing regimen [2]. A much larger success for a PLG microsphere product is Risperdal Consta ${ }^{\circledR}$. Risperidone is an anti-psychotic medicine used to treat schizophrenia and bipolar disorder. Administered as a daily oral medication, patient compliance is critically important as missed doses can lead to relapse and increased psychotic symptoms. In 2003, the FDA approved Risperdal Consta ${ }^{\circledR}$, a controlled release form of risperidone encapsulated in PLG microspheres that enabled dosing once every 2 weeks by intramuscular injection. Compared with the parent oral risperidone, less frequent dosing and improved compliance with administration by a health care professional rather than patient self-administration has made Risperdal Consta® a major success for treating these brain disorders [3].

\section{Advancing drug delivery with nanoparticles}

The many successes of controlled release microsphere-based medicines led innovators to explore the potential of drug delivery for systemic administration to reach specific sites of disease, with particular interest in targeting tumors for treating cancer. This cannot be achieved with microspheres, because they would be rapidly cleared from plasma. As a result, research efforts shifted toward reducing particle sizes (diameters $\leq 100 \mathrm{~nm}$ ) and surface modification using poly(ethylene glycol) to avoid immune recognition and clearance. Seminal papers in the field of nanomedicine were published in the early 1990s on PEGylated liposomes [4] and polymeric nanoparticles [5]. These efforts built upon the pioneering research by Maeda and Matsumura in the mid-1980s which demonstrated enhanced permeability and retention of macromolecules based upon their size owing to tumor leaky vasculature and poor lymphatic drainage [6].

The first FDA-approved cancer nanomedicine was Doxil®, a PEGylated liposome containing doxorubicin. It was initially approved in 1995 for the treatment of AIDSrelated Kaposi's sarcoma, and subsequently for treating ovarian cancer and multiple myeloma (in combination with bortezomib). Doxil increased doxorubicin circulation halflife by approximately 4-fold and tumor drug levels up to 10fold in preclinical models [4]. By containing doxorubicin within the liposome and targeting more drug to tumor, Doxil also showed an improved toxicity profile in patients, by greatly reducing the cardiovascular side effects of free doxorubicin [7].

Ten years after the initial approval of Doxil, a new nanoparticle-based cancer drug received its first approval. In 2005, Abraxane ${ }^{\circledR}$, a nanoparticle albumin-bound paclitaxel, was initially approved for treating breast cancer. It has also been approved for treating non-small cell lung and pancreatic cancers. Due to its poor solubility, paclitaxel (Taxol®) was originally developed by being dissolved in cremophor EL, a polyoxyethylated castor oil, which can lead to severe anaphylactic or hypersensitivity reactions. By solubilizing paclitaxel in albumin nanoparticles, Abraxane eliminated the need for cremophor and improved the tolerability profile of paclitaxel, with the highest label recommendation for Taxol at $175 \mathrm{mg} /$ $\mathrm{m}^{2}$, whereas Abraxane can be dosed as high as $260 \mathrm{mg} / \mathrm{m}^{2}$ [8].

\section{Enabling true therapeutic innovation with nanoparticles}

The first two waves of drug delivery enabled products with enhanced utility, compliance, and therapeutic window relative to the existing therapeutic agent. Current drug delivery innovations feature ambitious efforts to deliver large macromolecules to specific disease locations on timelines generally thought impossible. The developments of many of today's most innovative therapeutics are utilizing nanoparticle drug delivery systems, and for our scope, we will focus on RNA nanomedicines.

In 1998, Fire and Mello started a new revolution for gene therapy and gene editing, beginning with the statement, "Experimental introduction of RNA into cells can be used in certain biological systems to interfere with the function of an endogenous gene" [9]. Significant research has gone into developing short interfering RNA (siRNA) for RNA interference to turn off destructive biological pathways. Unmodified RNAs require drug delivery systems as they are not stable in biological fluids and do not readily penetrate into target cells where their action is required to reprogram the production of proteins. Building off the success of Doxil and other liposomal or lipid nanoparticles, major efforts focused on the optimization of lipid nanoparticles for the delivery of RNA [10].

Twenty years later in 2018, the first siRNA product was approved in 2018. Onpattro® (Patisiran) is a lipid nanoparticle containing siRNA to stop the toxic production of transthyretin in patients with polyneuropathies induced by hereditary transthyretin amyloidosis [11].

A second RNA wave has focused on developing messenger RNA (mRNA) therapies to promote healthy protein expression to treat diseases. A major potential advantage of mRNA therapies is the possibility to treat patients regardless of any 
particular genetic mutation. For cystic fibrosis, mutations in the cystic fibrosis $(\mathrm{CF})$ transmembrane conductance regulator (CFTR) gene can impact CFTR protein folding and capability to regulate salt transport. The most debilitating impact of $\mathrm{CF}$ and primary cause of mortality is the inability to clear mucus from the lungs, causing build-up and decreased lung function. Multiple programs, one of which is in early clinical development [12], are focused on inhaled mRNA to encode normal CFTR protein to restore healthy lung function. These approaches entail encapsulation of mRNA in lipid nanoparticles which are then inhaled as nebulized solutions to allow delivery to the upper airways where CFTR protein is produced.

In addition to therapeutic treatments, mRNA vaccines offer a promising opportunity due to their high potency, capacity for rapid development, and safe administration [13]. These potential advantages for mRNA vaccines are currently being played out in real time, as the Covid-19 pandemic is driving innovation and implementation in an effort to rapidly develop an mRNA vaccine. Several academic and industry groups around the world are putting a huge effort into developing therapeutics and vaccines for Covid-19. One company whose efforts merit highlighting at this point is Moderna. On January 11, 2020, the Chinese authorities shared the genetic sequence of the novel coronavirus. On January 13, 2020, the NIH Vaccine Research Center and Moderna's infectious disease research team finalized the sequence for the SARS-CoV-2 vaccine and Moderna mobilized toward clinical manufacture. The first clinical batch was completed on February 7, 2020, and underwent analytical testing; it was shipped on February 24, 2020, from Moderna and delivered to NIH from the Company's manufacturing facility in 42 days from sequence selection. The first participant in an NIH-led phase 1 clinical study was dosed on March 16, 2020 [14]. Such a timeline from virus sequencing to human dosing has never been achieved and was likely never thought possible. A critical enabling element to this impressive achievement was the development of a robust lipid nanoparticle delivery platform with the capability to be applied across mRNA sequences for developing vaccines as well as therapeutic mRNA modalities $[15,16]$.

\section{Conclusion}

From rather modest beginnings, drug delivery technologies are now enabling a vast landscape of new and innovative therapies. From academic labs to large biopharmaceutical companies, the collaboration between scientists, engineers, and clinicians will continue to advance therapies with drug delivery systems to address ongoing unmet patient needs. Over the past 50 years, we have witnessed an incredible evolution in drug delivery. Earlier generations featured improved versions of existing drugs utilizing controlled release microspheres and long-circulating nanoparticles for cancer treatments. Today, innovative nanotechnologies are core to the development of novel medicines to treat a range of serious diseases.

\section{References}

1. Langer R, Folkman J. Polymers for the sustained release of proteins and other macromolecules. Nature. 1976;263(5580):797-800.

2. FDA: Nutropin Depot prescribing information: https://www. accessdata.fda.gov/drugsatfda docs/label/2004/21075s008lbl.pdf

3. Lafeuille MH, Dean J, Carter V, Duh MS, Fastenau J, Dirani R, et al. Systematic review of long-acting injectables versus oral atypical antipsychotics on hospitalization in schizophrenia. Curr Med Res Opin. 2014;30(8):1643-55.

4. Gabizon A, et al. Prolonged circulation time and enhanced accumulation in malignant exudates of doxorubicin encapsulated in polyethylene-glycol coated liposomes. Cancer Res. 1994;54(4): 987-92.

5. Gref R, Minamitake Y, Peracchia MT, Trubetskoy V, Torchilin V, Langer R. Biodegradable long-circulating polymeric nanospheres. Science. 1994;263(5153):1600-3.

6. Matsumura Y, Maeda H. A new concept for macromolecular therapeutics in cancer chemotherapy: mechanism of tumoritropic accumulation of proteins and the antitumor agent smancs. Cancer Res. 1986;46:6387-92.

7. O'Brien MER, et al. Reduced cardiotoxicity and comparable efficacy in a phase III trial of pegylated liposomal doxorubicin $\mathrm{HCl}$ (CAELYXTM/Doxil) versus conventional doxorubicin for firstline treatment of metastatic breast cancer. Ann Oncol. 2004;15: 440-9.

8. Martín M. nab-Paclitaxel dose and schedule in breast cancer. Breast Cancer Res. 2015;17:81-91.

9. Fire A, Xu S, Montgomery MK, Kostas SA, Driver SE, Mello CC. Potent and specific genetic interference by double-stranded RNA in Caenorhabditis elegans. Nature. 1998;391(6669):806-11.

10. Allen TM, Cullis PR. Liposomal drug delivery systems: from concept to clinical applications. Adv Drug Deliv Rev. 2013;65(1):3648.

11. Akinc A, Maier MA, Manoharan M, Fitzgerald K, Jayaraman M, Barros S, et al. The Onpattro story and the clinical translation of nanomedicines containing nucleic acid-based drugs. Nat Nanotechnol. 2019;14(12):1084-7.

12. Translate bio: safety and tolerability of a single dose of MRT5005, a nebulized CFTR mRNA therapeutic, in adult CF patients. https:// investors.translate.bio/static-files/e1 ba9484-8a0b-40cf-9db9cbe2da9a51b9

13. Pardi N, Hogan MJ, Porter FW, Weissman D. mRNA vaccines-a new era in vaccinology. Nat Rev Drug Discov. 2018;17(4):261-79.

14. Moderna: Moderna announces first participant dosed in NIH-led phase 1 study of mRNA vaccine (mRNA-1273) against novel corona virus. https://investors.modernatx.com/news-releases/newsrelease-details/moderna-announces-first-participant-dosed-nih-ledphase-1-study

15. Hassett KJ, Benenato KE, Jacquinet E, Lee A, Woods A, Yuzhakov $\mathrm{O}$, et al. Optimization of lipid nanoparticles for intramuscular administration of mRNA vaccines. Mol Ther Nucleic Acids. 2019;15: $1-11$.

16. Sabnis S, Kumarasinghe ES, Salerno T, Mihai C, Ketova T, Senn JJ, et al. A novel amino lipid series for mRNA delivery: improved 
Endosomal escape and sustained pharmacology and safety in nonhuman primates. Mol Ther. 2018;26(6):1509-19.

Publisher's note Springer Nature remains neutral with regard to jurisdictional claims in published maps and institutional affiliations.

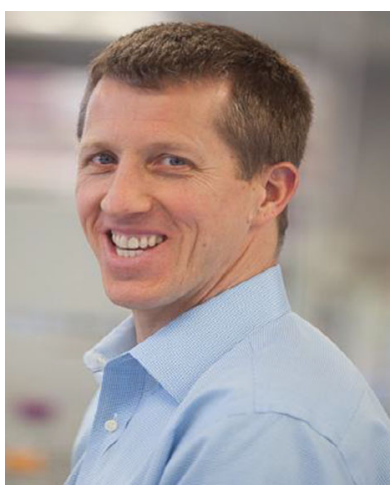

Jeff Hrkach, $\mathrm{PhD}$, is Senior Vice President of Technology Development at Frequency Therapeutics, leading all $\mathrm{CMC}$ and drug delivery efforts for their progenitor cell activation focused regenerative medicine pipeline. Prior to Frequency, Dr. Hrkach held senior R\&D leadership positions at Moderna, BIND Therapeutics, Momenta Pharmaceuticals, Alkermes and Advanced Inhalation Research (AIR).

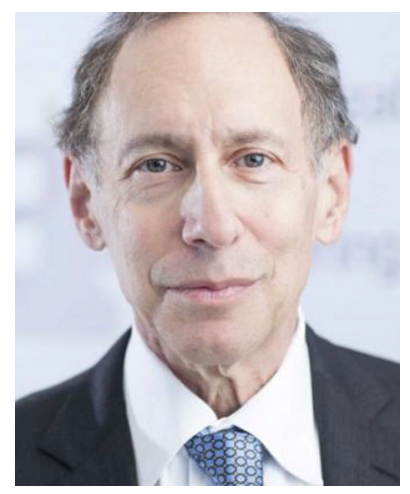

Robert S. Langer is one of 12 Institute Professors at MIT; being an Institute Professor is the highest honor that can be awarded to a faculty member. Dr. Langer has written approximately 1500 articles. He also has nearly 1400 issued and pending patents worldwide. Dr. Langer's patents have been licensed or sublicensed to over 400 pharmaceutical, chemical, biotechnology and medical device companies. He is the most cited engineer in history (h-index 273 with over 308,000 citations according to Google Scholar). 